\title{
Detection of turbulent thermal diffusion of particles in numerical simulations
}

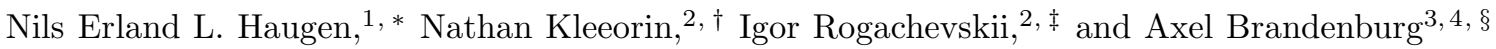 \\ ${ }^{1}$ SINTEF Energy Research, N-7034 Trondheim, Norway \\ ${ }^{2}$ Department of Mechanical Engineering, Ben-Gurion University of the Negev, P.O.Box 653, Beer-Sheva 84105, Israel \\ ${ }^{3}$ NORDITA, AlbaNova University Center, Roslagstullsbacken 23, SE 10691 Stockholm, Sweden \\ ${ }^{4}$ Department of Astronomy, Stockholm University, SE 10691 Stockholm, Sweden
}

(Dated: October 27, 2018, Revision: 1.177 )

\begin{abstract}
The phenomenon of turbulent thermal diffusion in temperature-stratified turbulence causing a non-diffusive turbulent flux of inertial and non-inertial particles in the direction of the turbulent heat flux is found using direct numerical simulations (DNS). In simulations with and without gravity, this phenomenon is found to cause a peak in the particle number density around the minimum of the mean fluid temperature for Stokes numbers less than 1, where the Stokes number is the ratio of particle Stokes time to turbulent Kolmogorov time at the viscous scale. Turbulent thermal diffusion causes the formation of large-scale inhomogeneities in the spatial distribution of inertial particles. The strength of this effect is maximum for Stokes numbers around unity, and decreases again for larger values. The dynamics of inertial particles is studied using Lagrangian modelling in forced temperature-stratified turbulence, whereas non-inertial particles and the fluid are described using DNS in an Eulerian framework.

PACS numbers: $47.27 . t b, 47.27 . T-$, 47.55.Hd, 47.55.Kf
\end{abstract}

\section{INTRODUCTION}

Transport and mixing of small particles (aerosols and droplets) in turbulent fluid flow is of fundamental importance in a large variety of applications (environmental sciences, physics of the atmosphere and meteorology, industrial turbulent flows and turbulent combustion; see, e.g., [1 [8]). There are also astrophysical applications, in particular in the context of protoplanetary accretion discs $[9-12]$.

Numerous laboratory [4, 13 17] and numerical 1824] experiments as well as observations in atmospheric $[6,8,25,26]$ and astrophysical [9 12, 27, 28] turbulent flows have shown different kinds of large-scale and smallscale long-living inhomogeneities (clusters) in the spatial distribution of particles. It is well known that turbulent diffusion causes destruction of large-scale inhomogeneities in the spatial distributions of particles. But how can we explain the opposite process resulting in a formation of large-scale clusters of particles?

One of the mechanisms of formation of particle inhomogeneities in temperature-stratified turbulence is the phenomenon of turbulent thermal diffusion [29]. This effect consists of a turbulent non-diffusive flux of inertial particles in the direction of the turbulent heat flux, so that particles are accumulated in the vicinity of the mean temperature minimum. The particular form of the flow field does not play any role in this effect. It is a purely collective phenomenon caused by temperature stratified turbulence resulting in a pumping effect,

\footnotetext{
* Nils.E.Haugen@sintef.no

$\dagger$ nat@bgu.ac.il

$\ddagger$ gary@bgu.ac.il

$\S$ brandenb@nordita.org
}

i.e., appearance of the non-zero mean effective velocity of particles in the direction opposite to the mean temperature gradient. A competition between two different phenomena, namely the turbulent thermal diffusion and the turbulent diffusion determines the conditions for the formation of large-scale particle clusters in the vicinity of the mean temperature minimum. The characteristic scale of the particle inhomogeneity formed due to the turbulent thermal diffusion is much larger than the integral scale of the turbulence. Furthermore, the characteristic time scale of the formation of the particle inhomogeneity is much longer than the characteristic turbulent time, i.e., this is a mean-field effect.

The phenomenon of turbulent thermal diffusion has been predicted theoretically in [29] and detected in different laboratory experiments in stably and unstably temperature-stratified turbulence 30 34]. This phenomenon is shown to be important for atmospheric turbulence with temperature inversions [35] and for smallscale particle clustering in temperature-stratified turbulence [34], but it is also expected to be significant for different kinds of heat exchangers, e.g., industrial boilers where Reynolds numbers and temperature gradients are large.

In spite of the fact that turbulent thermal diffusion has already been found in different types of laboratory experiments and atmospheric flows, this effect has never been observed in direct numerical simulations. The main goal of this paper is to find turbulent thermal diffusion of non-inertial and inertial particles in direct numerical simulations (DNS).

The paper is organized as follows. In Sect. II we discuss the physics of the phenomenon of turbulent thermal diffusion. The numerical simulations for fluid, inertial and non-inertial particles, and the results of direct numerical simulations are described in Sect. III. Motions 
of inertial particles are determined using a Lagrangian framework (Sections III-B,C,D), while non-inertial particles are described using an Eulerian framework (Sect. III-E). Conclusions are drawn in Sect. IV.

\section{PHYSICS OF TURBULENT THERMAL DIFFUSION}

In this section we discuss the physics of the phenomenon of turbulent thermal diffusion. The number density $n_{p}(t, \boldsymbol{r})$ of particles advected in a turbulent flow is determined by the following equation 36,37 ]

$$
\frac{\partial n_{p}}{\partial t}+\boldsymbol{\nabla} \cdot\left(n_{p} \boldsymbol{U}_{\mathrm{p}}-D \boldsymbol{\nabla} n_{p}\right)=0,
$$

where $D$ is the coefficient of Brownian diffusion of particles and $\boldsymbol{U}_{\mathrm{p}}$ is a random velocity of the particles which they acquire in a turbulent velocity field $\boldsymbol{U}$. In order to study the formation of large-scale inhomogeneous particle structures, Eq. (1) for the particle number density is averaged over an ensemble of turbulent velocity fields. This yields an equation for the mean number density, $N$, of particles that has been derived using different approaches in [29, 35, 38, 40]:

$$
\frac{\partial N}{\partial t}+\nabla \cdot\left[N\left(\boldsymbol{W}_{\mathrm{g}}+\boldsymbol{V}^{\mathrm{eff}}\right)-\left(D+D_{\mathrm{T}}\right) \boldsymbol{\nabla} N\right]=0,
$$

where

$$
\boldsymbol{V}^{\mathrm{eff}}=-\tau_{\mathrm{f}} \overline{\boldsymbol{U}_{\mathrm{p}}\left(\boldsymbol{\nabla} \cdot \boldsymbol{U}_{\mathrm{p}}\right)}=-\alpha D_{\mathrm{T}} \boldsymbol{\nabla} \ln \bar{T}
$$

is the effective pumping velocity of particles due to turbulent thermal diffusion, $\bar{T}$ is the mean fluid temperature, $\alpha$ is the turbulent thermal diffusion ratio (see below), $D_{\mathrm{T}}$ is the turbulent diffusion coefficient, $\tau_{\mathrm{f}}$ is the fluid turbulent integral timescale, overbars denote ensemble averaging, $\boldsymbol{W}_{\mathrm{g}}=\tau_{\mathrm{p}} \boldsymbol{g}$ is the terminal fall velocity of particles, $\boldsymbol{g}$ is the gravitational acceleration, $\tau_{\mathrm{p}}$ is the Stokes time that describes particle-fluid interactions. Equation (2) is written for a zero mean fluid velocity. For large Péclet numbers, the coefficient $\alpha$ is unity for non-inertial particles, while for inertial particles $\alpha$ depends on $\tau_{\mathrm{p}}$, the Mach number and the fluid Reynolds number [29, 35].

Already simple arguments allow us to estimate the effective particle velocity $\boldsymbol{V}^{\text {eff }}$. Let us estimate the turbulent flux of particles $\overline{n \boldsymbol{U}_{\mathrm{p}}}$. To this end we average Eq. (1) over an ensemble of turbulent velocity fields, and subtract the obtained averaged equation from Eq. (11). This yields an equation for the fluctuations of the particle number density, $n=n_{p}-N$ :

$$
\frac{\partial n}{\partial t}+\boldsymbol{\nabla} \cdot\left[n \boldsymbol{U}_{\mathrm{p}}-\overline{n \boldsymbol{U}_{\mathrm{p}}}-D \boldsymbol{\nabla} n\right]=-\boldsymbol{\nabla} \cdot\left(N \boldsymbol{U}_{\mathrm{p}}\right),
$$

where for simplicity we consider the case of zero mean particle velocity, $\overline{U_{\mathrm{p}}}=0$. The left hand side of Eq. (4) has dimension $n / \tau$, where the time $\tau$ can be identified with the fluid turbulent integral timescale $\tau_{\mathrm{f}}$ for large fluid Reynolds numbers. The fluctuations of particle number density can then be estimated as

$$
n \sim-\tau_{\mathrm{f}}\left(\boldsymbol{\nabla} \cdot \boldsymbol{U}_{\mathrm{p}}\right) N-\tau_{\mathrm{f}}\left(\boldsymbol{U}_{\mathrm{p}} \cdot \boldsymbol{\nabla}\right) N .
$$

Multiply Eq. (5) by $\boldsymbol{U}_{\mathrm{p}}$ and average this equation over an ensemble of turbulent velocity fields, we arrive at a formula for the turbulent flux of particles:

$$
\overline{n \boldsymbol{U}_{\mathrm{p}}}=N \boldsymbol{V}^{\mathrm{eff}}-\boldsymbol{D}_{T} \boldsymbol{\nabla} N,
$$

where the first term on the right hand side of Eq. (6) describes the turbulent flux of particles due to turbulent thermal diffusion: $N \boldsymbol{V}^{\mathrm{eff}}=-N \tau_{\mathrm{f}} \overline{\boldsymbol{U}_{\mathrm{p}}\left(\boldsymbol{\nabla} \cdot \boldsymbol{U}_{\mathrm{p}}\right)}$, and the second term on the right hand side of Eq. (6) determines the flux of particles caused by turbulent diffusion (see, e.g., [35]):

$$
\boldsymbol{D}_{T} \boldsymbol{\nabla} N=\tau_{\mathrm{f}} \overline{\left(\boldsymbol{U}_{\mathrm{p}}\right)_{i}\left(\boldsymbol{U}_{\mathrm{p}}\right)_{j}} \nabla_{j} N \approx \tau_{\mathrm{f}} \overline{U_{i} U_{j}} \nabla_{j} N,
$$

and $\boldsymbol{D}_{T}=\tau_{\mathrm{f}} \overline{U_{i} U_{j}}$ is the turbulent diffusion tensor. For isotropic turbulence $\boldsymbol{D}_{T}=D_{T} \delta_{i j}$, where $D_{T}=\tau_{\mathrm{f}} \overline{\boldsymbol{U}^{2}} / 3$.

\section{A. Inertial particles}

The non-diffusive mean flux of particles, $N \boldsymbol{V}^{\text {eff }}$, toward the mean temperature minimum is the main reason for the formation of large-scale inhomogeneous distributions of inertial particles in temperature-stratified turbulence. Indeed, the steady-state solution of Eqs. (2) and (3) for the mean number density of inertial particles is given by

$$
\tilde{N}(z)=[\tilde{T}(z)]^{-\alpha} \exp \left[-\int_{z_{0}}^{z}\left[W_{\mathrm{g}} / D_{\mathrm{T}}\right] \mathrm{d} z^{\prime}\right],
$$

where $W_{\mathrm{g}}=\left|\boldsymbol{W}_{\mathrm{g}}\right|$ is the modulus of the terminal fall velocity, $\tilde{T}=\bar{T} / \bar{T}_{0}$ is the non-dimensional mean fluid temperature, $T_{0}$ is the mean temperature far from the cooling zone, and $\tilde{N}=N / N_{0}$ is the non-dimensional mean number density of particles. Here the subscripts 0 represent the values at the boundary, and we neglect the small molecular flux of particles in Eq. (8) which describes the Brownian diffusion of particles. Equation (8) implies that small particles are accumulated in the vicinity of the mean temperature minimum.

In the following the mechanism of turbulent thermal diffusion for inertial particles with material density, $\rho_{\mathrm{p}}$, much larger than the fluid density, $\rho$, will be explained [29]. The inertia causes particles inside the turbulent eddies to drift out to the boundary regions between eddies. This can be seen by considering the equation of motion for particles, $\mathrm{d} \boldsymbol{U}_{\mathrm{p}} / \mathrm{d} t=-\left(\boldsymbol{U}_{\mathrm{p}}-\boldsymbol{U}\right) / \tau_{\mathrm{p}}$, where, for simplicity, the gravity force is neglected. The solution of the equation of motion for small Stokes time reads [41]:

$$
\boldsymbol{U}_{\mathrm{p}}=\boldsymbol{U}-\tau_{\mathrm{p}} \frac{\mathrm{d} \boldsymbol{U}}{\mathrm{d} t}+\mathrm{O}\left(\tau_{\mathrm{p}}^{2}\right)
$$


For large Reynolds numbers, this yields:

$$
\boldsymbol{\nabla} \cdot \boldsymbol{U}_{\mathrm{p}}=\boldsymbol{\nabla} \cdot \boldsymbol{U}+\frac{\tau_{\mathrm{p}}}{\rho} \nabla^{2} p+\mathrm{O}\left(\tau_{\mathrm{p}}^{2}\right)
$$

where $p$ is the fluid pressure. For large Péclet numbers, when molecular diffusion of particles in Eq. (11) can be neglected, it follows that $\boldsymbol{\nabla} \cdot \boldsymbol{U}_{\mathrm{p}} \propto-\mathrm{d} \ln n / \mathrm{d} t$. Therefore, in regions with maximum fluid pressure (i.e., where $\left.\nabla^{2} p<0\right)$, there is accumulation of inertial particles [i.e., $\left.\mathrm{d} n / \mathrm{d} t \propto-N\left(\tau_{\mathrm{p}} / \bar{\rho}\right) \nabla^{2} p>0\right]$. These regions have low vorticity, high strain rate, and maximum fluid pressure. Similarly, there is an outflow of inertial particles from regions with minimum fluid pressure.

In homogeneous and isotropic turbulence without mean gradients of temperature, a drift from regions with increased concentration of particles by a turbulent flow is equiprobable in all directions, and the pressure (temperature) of the surrounding fluid is not correlated with the turbulent velocity field. There is only non-zero correlation $\overline{(\boldsymbol{U} \cdot \boldsymbol{\nabla}) p}$ which contributes to the energy flux, while $\overline{\boldsymbol{U} p}=0$.

In temperature-stratified turbulence, temperature and velocity fluctuations are correlated due to a non-zero turbulent heat flux, $\overline{\boldsymbol{U} \theta} \neq \mathbf{0}$, where $\theta$ is the fluid temperature fluctuation. Fluctuations of temperature cause pressure fluctuations, which result in fluctuations of the number density of particles.

Increase of the pressure of the surrounding fluid is accompanied by an accumulation of particles, and the direction of the mean flux of particles coincides with that of the turbulent heat flux. The mean flux of particles is directed toward the minimum of the mean temperature, and the particles tend to be accumulated in this region [29, 35, 38]. To demonstrate that the directions of the mean flux of particles and the turbulent heat flux coincide, we assume that the mean temperature $T_{2}$ at point 2 is larger than the mean temperature $T_{1}$ at point 1 (see, e.g., 32]). We consider two small control volumes "a" and "b" located between these two points; see Table I. Let the direction of the local turbulent velocity in control volume "a" at some instant be the same as the

TABLE I. Mechanism of particle transport $\overline{n \boldsymbol{U}}$ for inertial particles along the direction of turbulent heat flux.

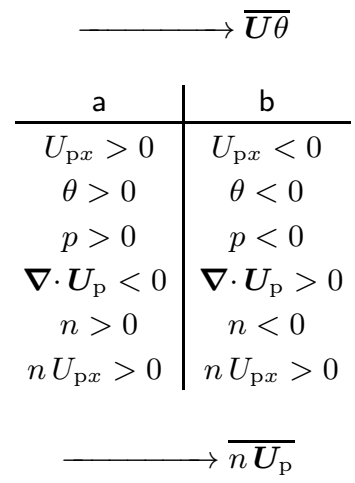

direction of the turbulent heat flux $\overline{\boldsymbol{U} \theta}$ (i.e., along the $x$ axis toward point 1) and let the local turbulent velocity in control volume "b", at the same instant, be directed opposite to the turbulent heat flux (i.e., toward point 2).

In temperature stratified turbulence, fluctuations of pressure $p$ and velocity $\boldsymbol{U}$ are correlated, and regions with a higher level of pressure fluctuations have higher temperature and velocity fluctuations. The fluctuations of temperature $\theta$ and pressure $p$ in control volume "a" are positive because $U_{\mathrm{p} x} \theta>0$, and negative in control volume "b"; see TableI. The fluctuations of particle number density $n$ are positive in control volume "a" [because particles are locally accumulated in the vicinity of the maximum of pressure fluctuations, $\mathrm{d} n / \mathrm{d} t \propto-N\left(\tau_{\mathrm{p}} / \bar{\rho}\right) \nabla^{2} p>$ $0]$, and they are negative in control volume " $\mathrm{b}$ " (because there is an outflow of particles from regions with low pressure). The flux of particles $n U_{x}$ is positive in control volume "a" (i.e., it is directed toward point 1 ), and it is also positive in control volume "b" (because both fluctuations of velocity and number density of particles are negative in control volume " $\mathrm{b}$ "). Therefore the mean flux of particles $\overline{n \boldsymbol{U}}$ is directed, as is the turbulent heat flux $\overline{\boldsymbol{U} \theta}$, toward point 1 . This causes the formation of large-scale inhomogeneous structures in the spatial distribution of inertial particles in the vicinity of the mean temperature minimum.

\section{B. Non-inertial particles}

Let us now consider non-inertial particles advected by the fluid flow such that the particle velocity coincides with the fluid velocity, $\boldsymbol{U}_{\mathrm{p}}=\boldsymbol{U}$. For low Mach numbers, using the anelastic approximation, $\boldsymbol{\nabla} \cdot \boldsymbol{U} \approx-\boldsymbol{U} \cdot \boldsymbol{\nabla} \ln \rho$, the effective velocity of non-inertial particles is given by

$$
V_{i}^{\mathrm{eff}}=-\tau_{\mathrm{f}} \overline{U_{i}(\boldsymbol{\nabla} \cdot \boldsymbol{U})}=\tau_{\mathrm{f}} \overline{U_{i} U_{j}} \nabla_{j} \ln \bar{\rho}
$$

where $\bar{\rho}$ is the mean fluid density. When $\boldsymbol{\nabla} \bar{p}=\mathbf{0}$ and anisotropy of turbulence is weak, the effective velocity of non-inertial particles is:

$$
\boldsymbol{V}^{\mathrm{eff}}=D_{\mathrm{T}} \boldsymbol{\nabla} \ln \bar{\rho}=-D_{\mathrm{T}} \boldsymbol{\nabla} \ln \bar{T},
$$

where $\bar{p}$ is the mean fluid pressure. The steady-state solution of Eqs. (2) and (12) for the mean number density of non-inertial particles is given by

$$
\tilde{N}(z)=[\tilde{\rho}(z)]^{D_{\mathrm{T}} /\left(D+D_{\mathrm{T}}\right)},
$$

where $\tilde{\rho}(z)=\bar{\rho} / \bar{\rho}_{0}$, where $\rho_{0}$ is the fluid density at the boundary.

It follows from Eq. (12) that the non-diffusive flux of particles is directed toward the maximum of the fluid density. The physics of the accumulation of the noninertial particles in the vicinity of the maximum of the mean fluid density (or the minimum of the mean fluid temperature) is quite different from that of inertial particles and can be explained as follows (see Table【I). Let us assume that the mean fluid density $\bar{\rho}_{2}$ at point 2 is larger 
TABLE II. Mechanism of particle transport $\overline{n \boldsymbol{U}}$ for noninertial particles along the direction of the mean fluid density gradient.

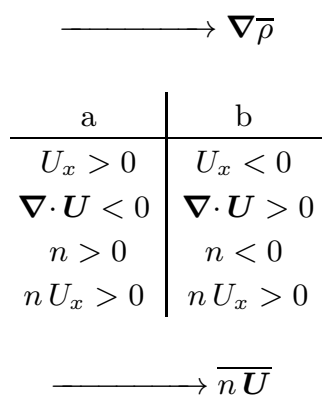

than the mean fluid density $\bar{\rho}_{1}$ at point 1 . Consider two small control volumes "a" and "b" located between these two points, and let the direction of the local turbulent velocity in control volume "a" at some instant be the same as the direction of the mean fluid density gradient $\nabla \bar{\rho}$ (i.e., along the $x$ axis toward point 2 ). Let the local turbulent velocity in control volume "b" be directed at this instant opposite to the mean fluid density gradient (i.e., toward point 1).

In a fluid flow with an imposed mean temperature gradient (i.e., an imposed mean fluid density gradient), one of the sources of particle number density fluctuations, $n \propto-\tau_{\mathrm{f}} N(\boldsymbol{\nabla} \cdot \boldsymbol{U})$, is caused by the non-zero $\boldsymbol{\nabla} \cdot \boldsymbol{U} \approx-\boldsymbol{U} \cdot \boldsymbol{\nabla} \ln \rho \neq 0$ [see the first term on the right hand side of Eq. (5)]. Since the fluctuations of the fluid velocity $\boldsymbol{U}$ are positive in control volume "a" and negative in control volume "b", we have $\boldsymbol{\nabla} \cdot \boldsymbol{U}<0$ in control volume "a", and $\boldsymbol{\nabla} \cdot \boldsymbol{U}>0$ in control volume "b". Therefore, the fluctuations of the particle number density $n \propto-\tau_{\mathrm{f}} N(\boldsymbol{\nabla} \cdot \boldsymbol{U})$ are positive in control volume "a" and negative in control volume "b". However, the flux of particles $n U_{x}$ is positive in control volume "a" (i.e., it is directed to point 2), and it is also positive in control volume "b" (because both fluctuations of fluid velocity and number density of particles are negative in control volume "b"). Therefore, the mean flux of particles $\overline{n \boldsymbol{U}}$ is directed, as is the mean fluid density gradient $\boldsymbol{\nabla} \bar{\rho}$, toward point 2 . This forms large-scale heterogeneous structures of non-inertial particles in regions with a mean fluid density maximum.

\section{DIRECT NUMERICAL SIMULATIONS}

\section{A. DNS model for the fluid}

In this study, equations for the fluid are solved by employing DNS in an Eulerian framework. All numerical simulations have been performed using the PENCIL CODE [42] (for details of the code, see Refs. [28, 43]). The set of compressible hydrodynamic equations is solved for the fluid density $\rho$, the fluid velocity $\boldsymbol{U}$, and the specific entropy $s$ :

$$
\begin{aligned}
& \frac{\mathrm{D} \ln \rho}{\mathrm{D} t}=-\boldsymbol{\nabla} \cdot \boldsymbol{U} \\
& \frac{\mathrm{D} \boldsymbol{U}}{\mathrm{D} t}=-\frac{1}{\rho}[\boldsymbol{\nabla} p-\boldsymbol{\nabla} \cdot(2 \rho \nu \mathbf{S})]+\boldsymbol{f}, \\
& T \frac{\mathrm{D} s}{\mathrm{D} t}=\frac{1}{\rho} \boldsymbol{\nabla} \cdot K \boldsymbol{\nabla} T+2 \nu \mathbf{S}^{2}-c_{\mathrm{P}}\left(T-T_{\mathrm{ref}}\right),
\end{aligned}
$$

where $T=T_{0} \exp \left(s / c_{\mathrm{V}}\right)\left(\rho / \rho_{0}\right)^{\gamma-1}$ is the fluid temperature, $\gamma=c_{\mathrm{P}} / c_{\mathrm{V}}$ is the ratio of specific heats at constant pressure and constant volume, respectively, $\mathrm{D} / \mathrm{D} t=$ $\partial / \partial t+\boldsymbol{U} \cdot \boldsymbol{\nabla}$ is the advective derivative, $\boldsymbol{f}$ is the external forcing function, $p=\left(c_{\mathrm{P}}-c_{\mathrm{V}}\right) \rho T$ is the fluid pressure, $\nu$ is the kinematic viscosity, and $K$ is the thermal conductivity. The traceless rate of strain tensor is given by

$$
\mathrm{S}_{i j}=\frac{1}{2}\left(U_{i, j}+U_{j, i}\right)-\frac{1}{3} \delta_{i j} \boldsymbol{\nabla} \cdot \boldsymbol{U},
$$

where $U_{i, j}=\nabla_{j} U_{i}$. The last term in the entropy equation (16) determines the cooling, which causes a temperature minimum at $z=0$, where

$$
T_{\text {ref }}=T_{0}+\delta T \exp \left(-z^{2} / 2 \sigma^{2}\right),
$$

$z$ is the vertical coordinate.

The size of the simulation domain is $L$ in all three directions. The smallest wavenumber in the domain, $k_{1}=2 \pi / L$. Wavenumbers are measured in units of $k_{1}$, and lengths in units of $k_{1}^{-1}$. The width of the cooling function is $\sigma=0.5 / k_{1}$. Furthermore, the strength of the cooling function, $\delta T$, is such that the relative contrast between temperature maximum and temperature minimum is $\sim 1.6$. In all simulations the Prandtl number $\operatorname{Pr}=\nu / K=1$.

Turbulence in the simulation box is produced by the forcing function $\boldsymbol{f}$, which is solenoidal and non-helical, i.e., $\boldsymbol{\nabla} \cdot \boldsymbol{f}=\boldsymbol{f} \cdot \boldsymbol{\nabla} \times \boldsymbol{f}=0$, and injects energy and momentum perpendicular to a random wavevector whose direction changes every timestep, but its length is approximately $k_{\mathrm{f}}$ (see [44]). The strength of the forcing is set such that the maximum Mach number is below 0.5. It has been checked that decreasing the Mach number by a factor of 5 does not have a significant effect on the results.

\section{B. Lagrangian model for inertial particles}

The equation of motion of the inertial particles is solved numerically in a Lagrangian framework. Particles are treated as point particles and we consider the oneway coupling approximation, i.e., there is an effect of the fluid on the particles only, while the particles do not influence the fluid motions. This is a good approximation when the spatial density of particles is much smaller than the fluid density. The particle equation of motion is

$$
\frac{\mathrm{d} \boldsymbol{U}_{\mathrm{p}}}{\mathrm{d} t}=\boldsymbol{g}-\frac{\boldsymbol{U}_{\mathrm{p}}-\boldsymbol{U}}{\tau_{\mathrm{p}}},
$$


where $\mathrm{d} \boldsymbol{X} / \mathrm{d} t=\boldsymbol{U}_{\mathrm{p}}$,

$$
\tau_{\mathrm{p}}=\frac{m_{p}}{3 \pi \mu d\left(1-f_{\mathrm{c}}\right)}
$$

is the Stokes time, $m_{p}=\rho_{\mathrm{p}}\left(\pi d^{3} / 6\right)$ is the particle mass of the spherical form, $d$ is the particle diameter, $\rho_{\mathrm{p}}$ is the particle material density, $\mu=\rho \nu$ is the dynamic viscosity, $f_{\mathrm{c}}=0.15 \mathrm{Re}_{\mathrm{p}}^{0.687}[3]$, and $\operatorname{Re}_{\mathrm{p}}=\left|\boldsymbol{U}_{\mathrm{p}}-\boldsymbol{U}\right| d / \nu$ is the particle Reynolds number. The key parameter of the problem is the Stokes number St $=\tau_{\mathrm{p}} / \tau_{\mathrm{k}}$ that is based on the particle Stokes time and the Kolmogorov timescale $\tau_{\mathrm{k}}=\tau_{\mathrm{f}} / \sqrt{\mathrm{Re}}$, where $\tau_{\mathrm{f}}=1 / u_{\mathrm{rms}} k_{\mathrm{f}}$ is the turbulent integral timescale, $\operatorname{Re}=u_{\mathrm{rms}} / \nu k_{\mathrm{f}}$ is the fluid Reynolds number and $u_{\mathrm{rms}}$ is the root mean square fluid velocity. The parameter $f_{\mathrm{c}}$ is used for inertial particles with $\operatorname{Re}_{\mathrm{p}} \geq 1$. Even though $f_{\mathrm{c}}$ is indeed used for calculating the particle forces in the simulations it is set to zero when calculating the Stokes number of a given particle. This is justified by the fact that for all particles considered here $f_{\mathrm{c}}$ is always very small and, furthermore, the Stokes number is only used for illustrative purposes. The ratio between the particle material density and the fluid mass density is $S=\rho_{\mathrm{p}} / \rho_{0}$, and for all simulations $S=1000$ outside the cooled zone.

To demonstrate robustness of the effect of particle accumulation in the vicinity of mean temperature minimum we use three different models for the dynamic viscosity $\mu$ : (i) $\mu=\mu_{*} \sqrt{T(z) / T_{0}}$, where $\mu_{*}=$ const; (ii) $\mu=$ const; (iii) $\mu=\rho(z) \nu$, where $\nu=$ const.

For most of the simulations the number of Eulerian grid points are $128^{3}$, while the number of Lagrangian particles is $5 \times 10^{5}$. It has been verified that increasing the number of grid points up to $512^{3}$ or the number of particles up to $10^{7}$ has no effect on the results.

In simulations without gravity, periodic boundary conditions are used in all directions both for the fluid and for the particles. When gravity is taken into account in the simulations, particles are made elastically reflecting from the vertical boundaries. It has been checked that adding small Brownian diffusion of particles does not affect the results.

In all our simulations, gravity is ignored in Eq. (15) for the fluid, but for the simulations presented in $\$$ IIIC it is included in Eq. (17) for particle motions. This situation has direct applications to atmospheric turbulence with lower-troposphere temperature inversions, where the density scale height due to gravity is about $8 \mathrm{~km}$, while the characteristic temperature inhomogeneity scale inside the temperature inversions is about $500-800 \mathrm{~m}$, and the integral scale of turbulence is about $50-100 \mathrm{~m}$ (see, e.g., [2]).

\section{Inertial particles in the absence of gravity}

Let us first discuss the results of the numerical simulations concerning the formation of the inertial particle inhomogeneities in the absence of gravity. In Figs. 1 and 2
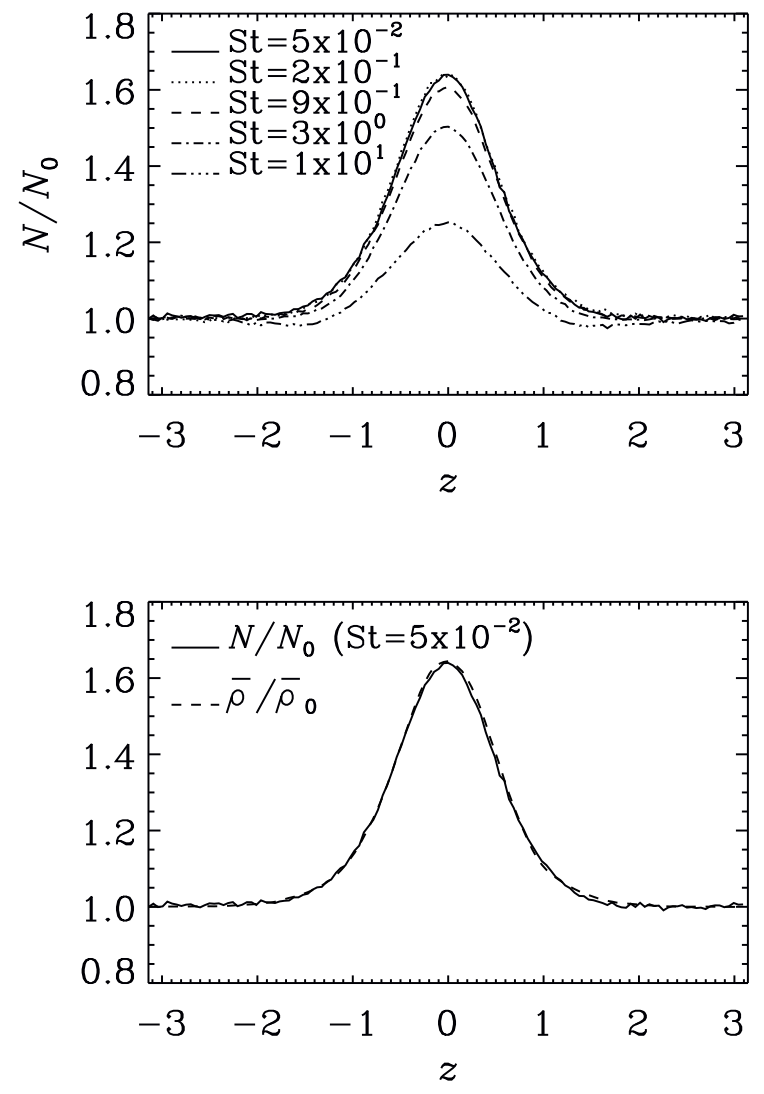

FIG. 1. Upper plot: vertical profile of the mean number density of inertial particles for different Stokes numbers for simulations with $k_{\mathrm{f}}=5 k_{1}, \mathrm{Re}=240$ in the case where the kinematic viscosity is kept constant and no gravity. Here the Stokes number St is based on the fluid density and temperature at the boundary. Lower plot: same as the upper plot, but showing the particle number density only for the smallest Stokes numbers together with the fluid mass density.

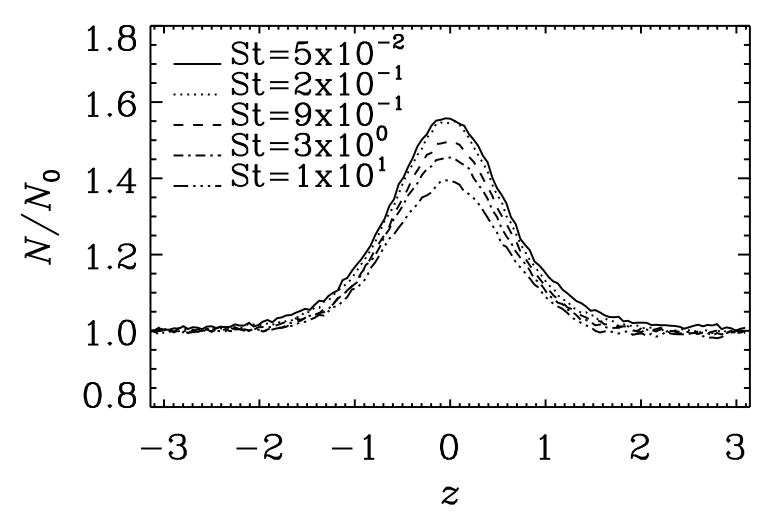

FIG. 2. The same as the upper plot of Fig. 1 but for the case where the dynamic viscosity is $\mu=\mu_{*} \sqrt{T(z) / T_{0}}, \mu_{*}$ is kept constant, and the Stokes number St is based on the fluid density and temperature at the boundary. 
we plot the vertical profile of the mean number density of particles for simulations with $k_{\mathrm{f}}=5 k_{1}, \mathrm{Re}=240$, different Stokes numbers, and different formulations for the dynamic viscosity. Inspection of these figures shows that, for a wide range of Stokes numbers, the maximum in the particle number density is located in the vicinity of the mean temperature minimum.

In the absence of gravity $\left(W_{\mathrm{g}}=0\right)$, the parameter $\alpha$ is given by $\alpha=-\ln \tilde{N} / \ln \tilde{T}$; see Eq. (8). In Fig. 3 the Stokes number dependence of the parameter $\alpha$ is shown for different Reynolds numbers and different forcing scales. It can be seen that the parameter $\alpha$ reaches its maximum value for $\mathrm{St}_{*} \approx 0.2$, while for Stokes numbers $\mathrm{St}>\mathrm{St}_{*}$, the value of $\alpha$ decreases monotonically. A critical Stokes number, $\mathrm{St}_{\mathrm{c}}$, separating the large and small Stokes number regimes might be defined such that $\alpha \geq 1$ for $\mathrm{St}<\mathrm{St}_{\mathrm{c}}$, while for $\mathrm{St}>\mathrm{St}_{\mathrm{c}}$ the parameter $\alpha$ is decreasing steeply with increasing Stokes number.

Since in the simulations the rms Mach number is not very small (around 0.2), the maximum value of the parameter $\alpha$ is only slightly larger than 1 . Indeed, the contribution of particle inertia to the coefficient $\alpha$ for small Stokes numbers is determined by $\boldsymbol{\nabla} \cdot \boldsymbol{U}_{\mathrm{p}} \propto$ $\left(\tau_{\mathrm{p}} / \bar{\rho}\right) \nabla^{2} p$. On the other hand, $\nabla^{2} p / \bar{\rho} \propto c_{\mathrm{s}}^{2} \nabla^{2} \theta / \bar{T} \propto$ $M^{-2} u_{\mathrm{rms}}^{2} \nabla^{2} \theta / \bar{T}$, where $M=u_{\mathrm{rms}} / c_{\mathrm{s}}$ is the Mach number, and $c_{\mathrm{s}}$ is the speed of sound. The deviation of the parameter $\alpha$ from 1 is of the order of [34]

$$
\alpha-1 \propto \frac{\tau_{\mathrm{p}}}{\tau_{\mathrm{f}}} M^{-2} .
$$

In this estimate we took into account that $\left|\overline{\boldsymbol{U} \nabla^{2} \theta}\right| \sim$ $\ell^{-2}|\overline{\boldsymbol{U} \theta}|$ and the turbulent heat flux is $\overline{\boldsymbol{U} \theta}=-\kappa_{T} \nabla \bar{T}$, where $\kappa_{T} \sim \tau_{\mathrm{f}} u_{\mathrm{rms}}^{2}$ and $\ell=\tau_{\mathrm{f}} u_{\mathrm{rms}}$.

Therefore, decreasing the Mach number causes $\alpha$ to increase due to particle inertia. For instance, in atmospheric turbulence the Mach number is of the order of $(0.1-3) \times 10^{-3}$ and $\alpha$ is about $10[35]$. On the other hand, the observed effect of accumulation of inertial particles occurs only for turbulent flows with large fluid Reynolds number. Decreasing the Mach number in the PENCIL CODE will either result in a shorter time step or a lower Reynolds number. This implies that we cannot easily reach at the same time small Mach numbers and large fluid Reynolds number. Therefore, the observations of the accumulation of inertial particles in the parameter range $\alpha \gg 1$ is not currently possible with the PENCIL Code.

The parameter $\alpha$ for the heavy particles decreases strongly with $\tau_{\mathrm{p}} \propto$ St (see Fig. 3 in the large Stokes number regime). Furthermore, it is expected that, in the large Stokes number regime, $\alpha$ is independent of Re for a particle as long as $u_{\mathrm{rms}}$ and $k_{\mathrm{f}}$ are kept constant. This is due to the fact that particles with $\tau_{\mathrm{p}} \sim \tau_{\mathrm{f}}$ are affected primarily by the largest turbulent eddies in the flow. This is because the smaller turbulent eddies have turnover times much shorter than the particle's Stokes time, and they cannot accelerate the particles. Since the

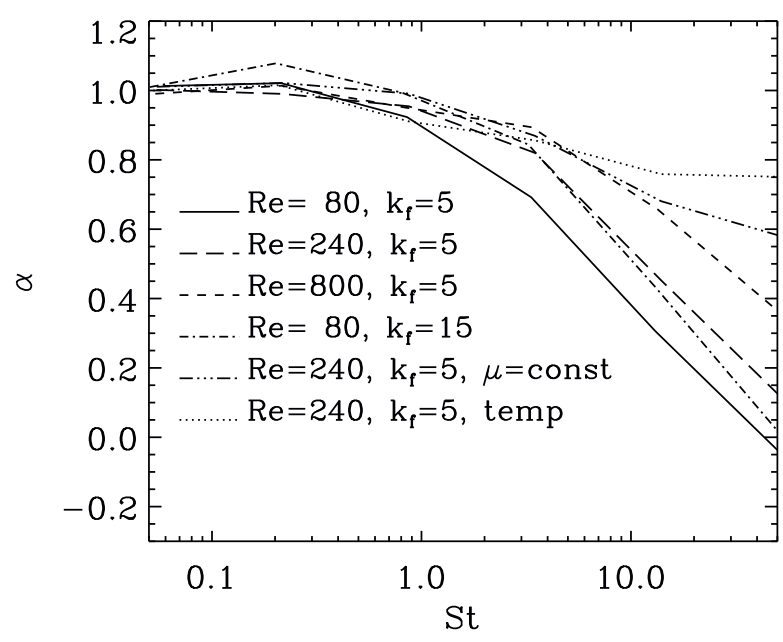

FIG. 3. The parameter $\alpha$ versus the Stokes number St for different Reynolds numbers and forcing wavenumbers in the absence of gravity. For the dashed-triple-dotted line the dynamic viscosity, $\mu$, is kept constant while for the dotted line named "temp" the dynamic viscosity $\mu=\mu_{*} \sqrt{T(z) / T_{0}}$, where $\mu_{*}$ is kept constant. For all other lines the dynamic viscosity, $\mu=\rho(z) \nu$, where the kinematic viscosity $\nu$ is kept constant. Here the Stokes number St is based on the values of fluid density and temperature at the boundary.

Stokes number is based on the Kolmogorov time $\tau_{\mathrm{k}}$, this implies that $\mathrm{St}_{\mathrm{c}} \propto \sqrt{\mathrm{Re}}$, which is indeed what is found in Fig. 3 .

When experiencing a high fluid density, a particle in the large Stokes number regime will more easily be accelerated by turbulence if the kinematic viscosity $\nu$ is constant than if the dynamic viscosity $\mu=\rho \nu$ is constant. This is due to the fact that for constant $\nu$ a high fluid density is associated with a smaller Stokes number, while for constant $\mu$ the Stokes number is independent of the fluid density. Due to this it is expected that particles with large Stokes numbers tend to be more depleted from the high density regions when $\nu$ is constant than when $\mu$ is constant. This explains why the simulation shown in Fig. 3 with constant $\mu$ has a much shallower fall-off with increasing Stokes number in the large Stokes number regime than the simulations with constant $\nu$.

If the integral scale of turbulence is close to the scale of the thermal cooling layer, a particle trapped inside a turbulent eddy might travel across the whole cooling zone during one eddy turnover time. This will effectively smear out the peak of the particle number density, and consequently also decrease the parameter $\alpha$. For simulations with $k_{\mathrm{f}}=5 k_{1}$, the integral scale is comparable to the cooling scale, and the peak of $\alpha$ for $k_{\mathrm{f}}=5 k_{1}$ is lower than for simulations with $k_{\mathrm{f}}=15 k_{1}$ (see Fig. 3). This trend is expected to continue for yet larger $k_{\mathrm{f}}$.

In addition, Fig. 3 shows that the difference in the parameter $\alpha$ determined for different formulations of the 
TABLE III. The coefficient $\alpha$, the relative maximum of the particle number density and profile width for simulations with $k_{\mathrm{f}}=5 k_{1}, \operatorname{Re}=240$ and for different formulations of the dynamic viscosity: (i) in the model $f_{1}$ the dynamic viscosity is $\mu=\mu_{*} \sqrt{T(z) / T_{0}}$, where $\mu_{*}$ is constant; (ii) in the model $f_{2}$ the dynamic viscosity is constant; (iii) in the model $f_{3}$ the dynamic viscosity $\mu=\rho(z) \nu$, where the kinematic viscosity $\nu$ is constant. The profile width $L_{N}$ is given at the level when the number density is decreased in $e$ times.

$$
\begin{array}{c|ccc|ccc|ccc} 
& \multicolumn{4}{|c}{\alpha} & \multicolumn{4}{c}{N_{\max } / N_{0}} & \multicolumn{3}{c}{\text { Profile width }} \\
\mathrm{St} & f_{1} & f_{2} & f_{3} & f_{1} & f_{2} & f_{3} & f_{1} & f_{2} & f_{3} \\
\hline 0.1 & 1.00 & 1.01 & 1.00 & 1.55 & 1.66 & 1.63 & 0.88 & 0.80 & 0.79 \\
1 & 0.91 & 0.98 & 0.95 & 1.49 & 1.62 & 1.60 & 0.86 & 0.80 & 0.79 \\
10 & 0.80 & 0.75 & 0.59 & 1.42 & 1.44 & 1.35 & 0.90 & 0.82 & 0.81 \\
14 & 0.76 & 0.68 & 0.45 & 1.39 & 1.39 & 1.25 & 0.90 & 0.83 & 0.82
\end{array}
$$

dynamic viscosity $\mu$, is very small for St $\ll 1$, and it is small for $\mathrm{St} \sim 1$. The real difference in the parameter $\alpha$ is only observed for large Stokes numbers, St $>10$ (see TABLE III). In this case, the largest value of $\alpha$ occurs for the dynamic viscosity $\mu=\mu_{*} \sqrt{T(z) / T_{0}}$, where $\mu_{*}=$ const. On the other hand, the difference in relative maximum of the particle number density and the profile width for different formulations of dynamic viscosity is very minor (see TABLE III). Also scale-separation increases effect of the particle accumulation for $\mathrm{St}>1$ [see Fig. 3. However, using different parameters and different formulations of the dynamic viscosity, we always observe the effect of particle accumulation in the vicinity of the mean temperature minimum. This is an indication of the robustness of this phenomenon.

\section{Inertial particles with gravity}

For heavy inertial particles with large Stokes numbers, gravity plays a crucial role, and must be included in the simulations. In Fig. 4, the mean particle number density is shown as a function of vertical position $z$ for simulations with $\mathrm{St}=1, k_{\mathrm{f}}=5 k_{1}$, and different gravitational accelerations.

In the following, gravity is measured in the units of $g_{0}=D_{\mathrm{T}} /\left(\tau_{\mathrm{k}} L\right)$, so that $g / g_{0}=L / L_{\mathrm{g}}$ for a particle of Stokes number unity. Here, $L_{\mathrm{g}}=D_{\mathrm{T}} / W_{\mathrm{g}}$ is the characteristic scale of the mean particle number density variations due to the gravity for an isothermal case, $D_{\mathrm{T}}=u_{\mathrm{rms}} / 3 k_{\mathrm{f}}$ is the turbulent diffusion coefficient, and $L$ is the height of the box.

As the particle sedimentation velocity is increased, the particle number density profile is more and more tilted, as expected. For large particle sedimentation velocity $\left(g / g_{0}=200 / 3\right)$, almost all particles have accumulated at the lower wall of the box (see Fig. 4).

In Fig. 5, the vertical profile of the particle number

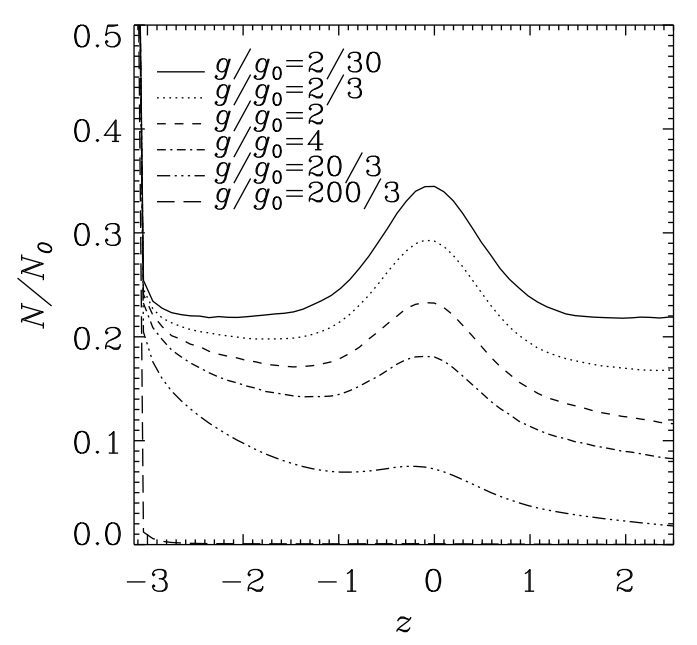

FIG. 4. Vertical profile of the mean number density for different gravitational accelerations, $\mathrm{St}=1$ and $k_{\mathrm{f}}=5 k_{1}$, where the kinematic viscosity $\nu$ is kept constant.

density is shown for $g / g_{0}=2 / 3$ and different Stokes numbers. By fitting these results with Eq. (8), the parameter $\alpha$ is found to be about 1 for the three smallest Stokes numbers in Fig. [5, while for $\mathrm{St}=3.5$ and $\mathrm{St}=14 \mathrm{a}$ best fit of $\alpha$ is found around 0.8 and 0.5 , respectively. For large Stokes numbers the maximum particle number density is found near the bottom wall of the box due to the large sedimentation velocity.

\section{E. Non-inertial particles}

For comparison with the numerical simulations performed for inertial particles in a Lagrangian framework, we describe in this section numerical simulations for noninertial particles, where the equations for both, fluid and particles are solved by employing DNS in an Eulerian framework. In particular, we now solve Eq. (1) for the number density of non-inertial particles $n_{p}$ with $\boldsymbol{U}_{p}=\boldsymbol{U}$, and the fluid density $\rho$, the fluid velocity $\boldsymbol{U}$, and the specific entropy $s$, using again the PencIL Code [42]. For these simulations the resolution is $128^{3}$. For the fluid we apply the same conditions as was described in previous subsections. In particular, the periodic boundary conditions are used in these simulations in three directions for Eqs. (1) and (14)-(16).

Non-inertial particles are characterized by the following dimensionless parameters: $\mathrm{Pe}=u_{\mathrm{rms}} / D k_{\mathrm{f}}$ is the Péclet number and $\mathrm{Sc}=\nu / D$ is the Schmidt number. The results of the simulations, shown in Fig. 6, demonstrate the accumulation of non-inertial particles in the vicinity of the maximum of the mean fluid density (or the minimum of the mean fluid temperature). This is in agreement with the theoretical predictions [29].

The results shown in Fig. 6] demonstrate that the pro- 

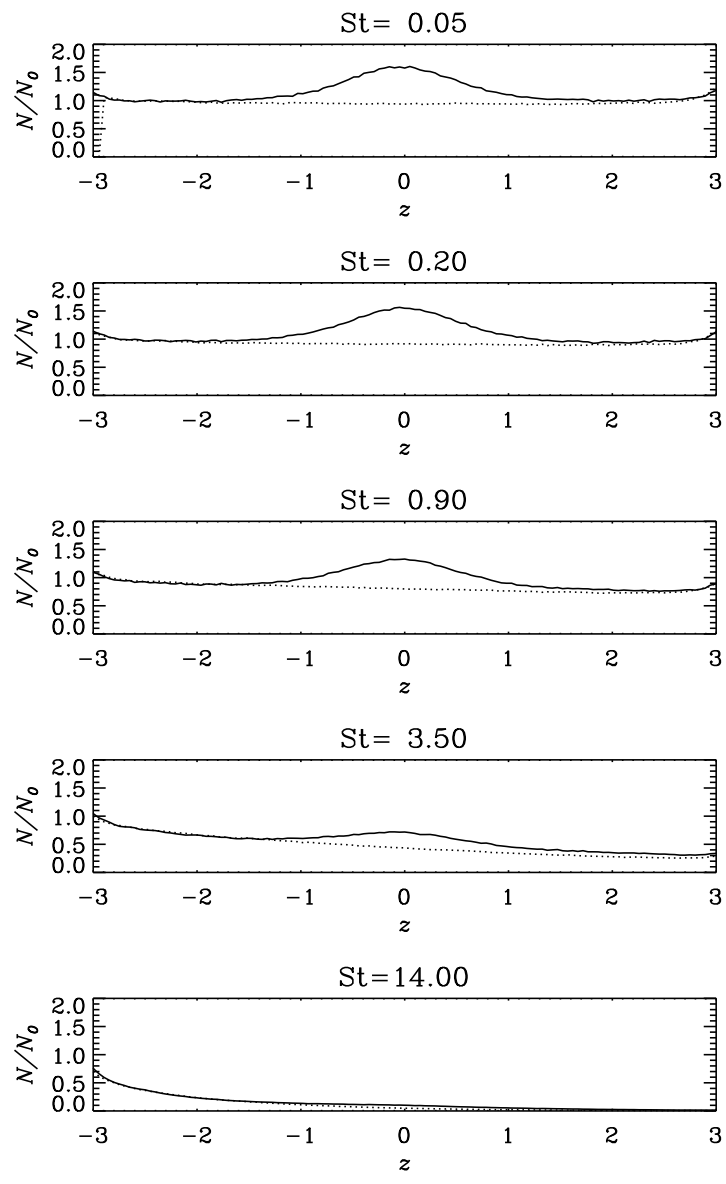

FIG. 5. Vertical profile of the mean number density (solid line) for $g / g_{0}=2 / 3, k_{\mathrm{f}}=5 k_{1}$ and different Stokes numbers which are based on the fluid density and temperature at the boundary. Here the kinematic viscosity $\nu$ is kept constant. The dotted line represent the isothermal reference case.

files of the mean fluid density and the mean particle number density are similar in numerical simulations with large Péclet and Reynolds numbers. However, when the Péclet number, $\mathrm{Pe}=\mathrm{ReSc}$ is not large, the molecular diffusion $D$ becomes important, and the profiles of the mean fluid density and the mean particle number density are, according to Eq. (13), no longer similar.

\section{CONCLUSIONS}

This study is the first numerical demonstration of the existence of the phenomenon of turbulent thermal diffusion of inertial and non-inertial particles in forced, temperature stratified turbulence. The inertial particles are described using a Lagrangian framework, while noninertial particles and the fluid flow are determined using an Eulerian framework. The phenomenon of turbulent thermal diffusion has been studied for different Stokes and fluid Reynolds numbers, Péclet numbers as well as different forcing scales of the turbulence. Furthermore,
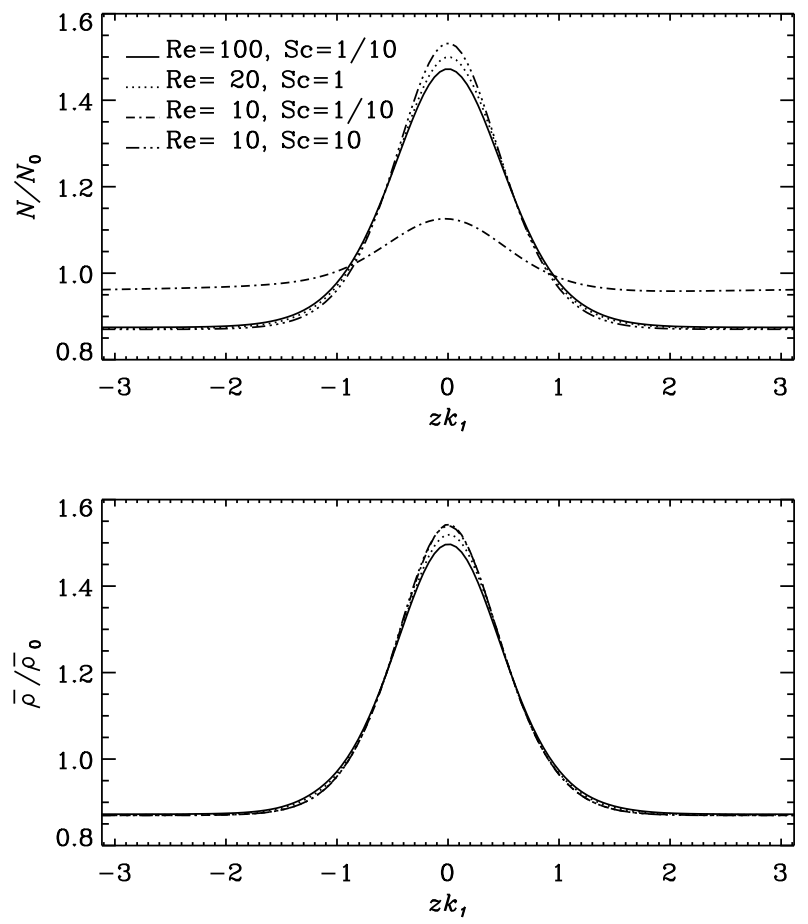

FIG. 6. Vertical profiles of the mean number density (upper panel) and the mean fluid density (lower panel) of non-inertial particles for simulations with $k_{\mathrm{f}}=5 k_{1}$ and different Reynolds and Schmidt numbers.

the effect of gravity has been included in simulations.

In all simulations, with different parameters and different formulations of the dynamic viscosity, we always observe the effect of particle accumulation in the vicinity of the mean temperature minimum due to turbulent thermal diffusion for St $<1$. This effect is robust and the results of the numerical simulations are in agreement with theoretical studies [29, 38], laboratory experiments 31, 33, 34] and atmospheric observations [35]. When St $>1$, this effect is decreasing with Stokes number.

\section{ACKNOWLEDGMENTS}

This work was supported in part by the Norwegian Research Council project PAFFrx (186933) (NELH), by COST Action MP0806 (IR), and by the European Research Council under the AstroDyn Research Project 227952 (AB). The authors acknowledge the hospitality of NORDITA. 
[1] G. T. Csanady, Turbulent Diffusion in the Environment (Reidel, Dordrecht, 1980).

[2] A. K. Blackadar, Turbulence and Diffusion in the Atmosphere (Springer, Berlin, 1997).

[3] C. T. Crowe, J. D. Schwarzkopf, M. Sommerfeld and Y. Tsuji, Multiphase flows with droplets and particles, second edition (CRC Press LLC, NY, 2011).

[4] Z. Warhaft, Annu. Rev. Fluid Mech. 32, 203 (2000); Fluid Dyn. Res. 41, 011201 (2009).

[5] S. Balachandar and J. K. Eaton, Annu. Rev. Fluid Mech. 42, 111 (2010).

[6] R. A. Shaw, Annu. Rev. Fluid Mech. 35, 183 (2003).

[7] R. E. Britter and S. R. Hanna, Annu. Rev. Fluid Mech. 35, 469 (2003).

[8] A. Khain, M. Pinsky, T. Elperin, N. Kleeorin, I. Rogachevskii and A. Kostinski, Atmosph. Res. 86, 1 (2007).

[9] V. S. Safronov, Evolution of the Protoplanetary Cloud and Formation of Earth and the Planets, (NASA Tech. Transl. F-677, Jerusalem: Israel Sci. Transl., 1972).

[10] T. Takeuchi, In: Lecture Notes in Physics "Small Bodies in Planetary Systems" (Springer, Berlin, 2009), Vol. 758, p. 1.

[11] L. S. Hodgson and A. Brandenburg, Astron. Astrophys. 330, 1169 (1998).

[12] A. Johansen, A. C. Andersen and A. Brandenburg, Astron. Astrophys. 417, 361 (2004).

[13] A. Aliseda, A. Cartellier, F. Hainaux and J. C. Lasheras, J. Fluid Mech. 468, 77 (2002).

[14] A. M. Wood, W. Hwang and J. K. Eaton, Int. J. Multiphase Flow 31, 1220 (2005).

[15] J. Salazar, J. de Jong, L. Cao, S. Woodward, H. Meng and L. Collins, J. Fluid Mech. 600, 245 (2008).

[16] H. Xu and E. Bodenschatz, Physica D 237, 2095 (2008).

[17] E. W. Saw, R. A. Shaw, S. Ayyalasomayajula, P. Y. Chuang and A. Gylfason, Phys. Rev. Lett. 100214501 (2008).

[18] G. Boffetta, F. De Lillo and A. Gamba, Phys. Fluids 16, L20 (2003).

[19] J. Chun, D. L. Koch, S. L. Rani, A. Ahluwalia and L. R. Collins, J. Fluid Mech. 536, 219 (2005).

[20] L. Chen, S. Goto and J. C. Vassilicos, J. Fluid Mech. 553, 143 (2006).

[21] H. Yoshimoto and S. Goto, J. Fluid. Mech. 577, 275 (2007).

[22] M. van Aartrijk and H. J. H. Clercx, Phys. Rev. Lett. 100, 254501 (2008).
[23] J. Bec, L. Biferale, M. Cencini, A. Lanotte, S. Musacchio and F. Toschi, Phys. Rev. Lett. 98, 084502 (2007).

[24] J. Bec, L. Biferale, A. Lanotte, A. Scagliarini and F. Toschi, J. Fluid Mech. 645, 497 (2010).

[25] P. A. Vaillancourt and M. K.Yau, Bull. Am. Met. Soc. 81, 2851 (2000).

[26] H. Siebert, S. Gerashchenko, A. Gylfason, K. Lehmann, L. R. Collins, R. A. Shaw and Z. Warhaft, Atmosph. Res. 97, 426 (2010).

[27] A. Johansen and A. Youdin, Astrophys. J. 662, 627 (2007).

[28] A. Johansen, J. S. Oishi, M. M. Mac Low, H. Klahr, T. Henning and A. Youdin, Nature 448, 1022 (2007).

[29] T. Elperin, N. Kleeorin and I. Rogachevskii, Phys. Rev. Lett. 76, 224 (1996); Phys. Rev. E 55, 2713 (1997).

[30] A. Eidelman, T. Elperin, N. Kleeorin, A. Krein, I. Rogachevskii, J. Buchholz, and G. Grünefeld, Nonl. Proc. Geophys. 11, 343 (2004).

[31] J. Buchholz, A. Eidelman, T. Elperin, G. Grünefeld, N. Kleeorin, A. Krein, I. Rogachevskii, Experim. Fluids 36, 879 (2004).

[32] A. Eidelman, T. Elperin, N. Kleeorin, A. Markovich, I. Rogachevskii, Nonl. Proc. Geophys. 13, 109 (2006).

[33] A. Eidelman, T. Elperin, N. Kleeorin, I. Rogachevskii and I. Sapir-Katiraie, Experim. Fluids 40, 744 (2006).

[34] A. Eidelman, T. Elperin, N. Kleeorin, B. Melnik and I. Rogachevskii, Phys. Rev. E 81, 056313 (2010).

[35] M. Sofiev, V. Sofieva, T. Elperin, N. Kleeorin, I. Rogachevskii and S. S. Zilitinkevich, J. Geophys. Res. 114, D18209 (2009).

[36] S. Chandrasekhar, Rev. Modern Phys. 15, 1 (1943).

[37] A. I. Akhiezer and S. V. Peletminsky, Methods of Statistical Physics (Pergamon, Oxford, 1981).

[38] T. Elperin, N. Kleeorin, I. Rogachevskii and D. Sokoloff, Phys. Rev. E 61, 2617 (2000); ibid 64, 026304 (2001).

[39] R. V. R. Pandya and F. Mashayek, Phys. Rev. Lett. 88, 044501 (2002).

[40] M. W. Reeks, Int. J. Multiph. Flow 31, 93 (2005).

[41] M. R. Maxey, J. Fluid Mech. 174, 441 (1987).

[42] The PEncIl CODE is a high-order finite-difference code (sixth order in space and third order in time); http://pencil-code.googlecode.com

[43] N. E. L. Haugen and S. Kragset, J. Fluid Mech. 661, 239-261 (2010).

[44] N. E. L. Haugen and A. Brandenburg, Phys. Fluids 18, 075106 (2006). 